\title{
Síndrome de burnout y clima social familiar en docentes de nivel inicial del distrito de Chincha Alta \author{
of the district of Chincha Alta
} \\ Burnout syndrome and family social climate in teachers of the initial level
}

Cecilia Sifuentes-Pachas ${ }^{1}$

\begin{abstract}
Resumen
Objetivo: determinar la relación entre el síndrome de burnout y el clima social familiar en las docentes del nivel inicial del distrito de Chincha Alta. Materiales y métodos: estudio correlacional de diseño no experimental - transversal. La muestra estuvo conformada por 141 docentes mujeres del nivel inicial. Las variables principales fueron el síndrome de Burnout para profesores (SB) y el clima social familiar, medidas a través del Maslach Burnout Inventory (MBI) y la Escala de Clima Social Familiar (FES). Se utilizó el paquete estadístico STATA 13, se realizó la prueba de normalidad de Kolmogorov Smirnov y para el análisis bivariado se utilizó U de Mann Whitney, ANOVA, Pearson y Spearman. Resultados: la muestra presentó una media de edad de 45.4 años, se encontró que existe asociación entre la dimensión relación de la dimensión familiar y el agotamiento emocional $(\mathrm{p}=0.04)$, entre las dimensiones de desarrollo $(\mathrm{p}=0.04)$, estabilidad $(\mathrm{p}=0.02)$ y el estado civil $(\mathrm{p}=0.05)$ con la dimensión de baja realización personal y la dimensión de relación con el turno ( $\mathrm{p}=0.05$ ). Conclusión: los niveles de desgaste emocional se asocian con los niveles de interacción dentro de la familia. Es importante que los profesionales de Psicología implementen programas preventivos promocionales a fin de mejorar los niveles óptimos del desarrollo integral de las docentes.
\end{abstract}

Palabras clave: Burnout; Agotamiento emocional; Despersonalización; Docentes (Fuente: DeCS).

\begin{abstract}
Objective: to determine the relationship between the burnout syndrome and the family social climate in the teachers of the initial level of the district of Chincha Alta. Materials and methods: a correlational study of non-experimental design - transversal. The sample consisted of 141 female teachers of the initial level. The main variables were the Burnout syndrome for teachers (SB) and the family social climate, measured through the Maslach Burnout Inventory (MBI) and the Family Social Climate Scale (FES). The statistical package STATA 13 was used, the Kolmogorov Smirnov normality test was carried out, and U of Mann Whitney, ANOVA, Pearson, and Spearman were used for the bivariate analysis. Results: the sample presented a mean age of 45.4 years, it was found that there is an as sociation between the relationship dimension of the family dimension and emotional exhaustion $(p=0.04)$, between the development dimensions $(p=0.04)$, stability $(p=0.02)$ and the marital status $(p=0.05)$ with the dimension of low personal fulfillment and the dimension of relationship with the shift $(\mathrm{p}=0.05)$. Conclusion: the levels of emotional exhaustion are associated with levels of interaction within the family. It is important that Psychology professionals implement promotional preventive programs in order to improve the optimal levels of the integral development of the teachers.
\end{abstract}

Keys words: Burnout; Emotional exhaustion; Depersonalization; Teachers (Source: DeCS).

Para citar:

Sifuentes C. Síndrome de burnout y clima social familiar en docentes de nivel inicial del distrito de Chincha Alta. CASUS. 2019;4(1):15-21.

${ }^{1}$ Puesto Parroquial de Salud Mental San José. Bachiller de Psicología. Corren electrónico: ceciliasifuentesp@gmail.com
Fecha de recepción: 05-02-19

Fecha de envío a pares: 08-02-19

Fecha de aprobación por pares: 11-04-19

Fecha de aceptación: 12-04-19 


\section{INTRODUCCIÓN}

Actualmente diversas investigaciones han evidenciado el aumento del síndrome de burnout en personas con labores de asistencia (1). Entre ellos destacan los profesionales de la docencia por la acumulación de actividades y funciones a realizar (1). Para la Organización Internacional del Trabajo a nivel mundial el estrés no es considerado un trastorno que perjudica a la salud, sino el primer signo o respuesta a un daño físico $\mathrm{y}$ emocional, que ocasiona un desequilibrio entre las exigencias, capacidades y los recursos percibidos de un trabajador para hacerles frente $(2,3)$. Sin embargo, en ocasiones no se limita a un signo, pues puede traer consecuencias mayores como el síndrome de burnout o síndrome del quemado que se manifiesta en un desgaste emocional, una actitud negativa hacia los demás o una evaluación del propio trabajo por debajo de los estándares (4).

El síndrome de burnout es una respuesta psicológica que implica un estado de agotamiento emocional, físico y psíquico producto de la conmoción negativa y acumulada de estrés laboral por tiempo prolongado $(1,5)$. En el ámbito laboral las personas buscan la realización de forma rápida lo cual puede ser agotador. Esta exigencia no sólo se evidencia en el trabajo, sino también en el entorno familiar. Muchos profesionales son, a su vez, miembros activos de un hogar por lo que se sobrecargan de responsabilidades provenientes de ambos contextos lo que repercute en su calidad de vida (6).

A nivel nacional, según el Instituto de Opinión Pública el $38.8 \%$ de los peruanos, tanto de la zona urbana como rural, mencionan haber tenido en los dos últimos años estrés (7). Por su parte, en la región Ica el $83.6 \%$ y en el distrito de Chincha Alta el $78.2 \%$ de los docentes han manifestado estrés (8). Paralelamente al empeño educativo y desarrollo profesional, algunos tienen un desempeño sindical (9). Respecto a la jornada laborativa el Ministerio de Educación del Perú (10) solicita un mínimo de 5 horas diarias de trabajo pedagógico, 25 horas semanales y 900 horas durante el año lectivo. La misma comprende, además de la elaboración de sesiones de clases y materiales didácticos, la participación grupos de inter aprendizaje de forma colegiada, tareas extracurriculares asignadas para el logro de los compromisos de la gestión escolar, organizar los talleres de escuela de padres, entre otras actividades (5). Sumado a lo anterior, a lo largo del ejercicio de la carrera, el profesor peruano suele mostrar diversas dificultades en su crecimiento profesional, asociadas a su posición socioeconómica. Estos elementos pueden resultar en una opresión dentro de su trabajo que suele expresarse en niveles de estrés altos. Los cuales, si se mantienen en el tiempo, pueden desembocar en una mengua de su función pedagógica (5).

Considerando lo anterior profundizar en la relación entre síndrome de burnout y clima social familiar en docentes es relevante, pues los problemas de salud mental de las mismas con frecuencia suelen presentarse como síntoma del malestar interior (7). La población de docentes a nivel inicial es mayoritariamente conformada por el sexo femenino (8). Muchas cumplen el doble rol de madres y amas de casa. Siendo la sobrecarga aún mayor por ejercer funciones profesionales. Se debe considerar, que parte del tiempo que le brindan a sus familias es usualmente compartida con las obligaciones en el trabajo (11). Esta difícil compatibilización entre la vida laboral y del hogar suele ser reflejada por algunas mediante la somatización y respuestas fisiológicas como dolores de cabeza, tensión frecuente, agotamiento, dolores musculares, entre otros (11). Asimismo, repercute a nivel psicológico puesto que las maestras ante la presión laboral y familiar llegan a tener desbalances a este nivel.

Por lo anteriormente mencionado, el objetivo fue determinar la relación entre el síndrome de burnout y clima social familiar en las docentes del nivel inicial del distrito de Chincha Alta.

\section{MATERIALES Y MÉTODOS}

Se realizó un estudio correlacional de corte transversal. La muestra estuvo conformada por 141 docentes de sexo femenino de 27 colegios estatales de nivel inicial pertenecientes al distrito de Chincha Alta; el muestreo fue no probabilístico 
por conveniencia. Se incluyó a docentes nombradas y contratadas que se encontraban ejerciendo la profesión en colegios públicos que aceptaron participar al estudio.

Tabla 1. Descripción de la muestra

\begin{tabular}{|c|c|c|}
\hline & $\mathbf{n}$ & $\%$ \\
\hline Edad $($ media \pm DS $)$ & \multicolumn{2}{|c|}{$45.4 \pm 9.2$} \\
\hline \multicolumn{3}{|l|}{ Estado Civil } \\
\hline Soltero & 28 & 19.9 \\
\hline Conviviente & 12 & 8.5 \\
\hline Casado & 82 & 58.2 \\
\hline Separado & 9 & 6.4 \\
\hline Viudo & 2 & 1.4 \\
\hline Divorciado & 8 & 5.7 \\
\hline \multicolumn{3}{|l|}{ Relaciones Personales } \\
\hline Con pareja estable & 99 & 70.2 \\
\hline Sin pareja estable & 42 & 29.8 \\
\hline \multicolumn{3}{|l|}{ Número de hijos } \\
\hline Sin hijos & 13 & 9.2 \\
\hline Con 1 hijo & 32 & 22.7 \\
\hline Más de 1 hijo & 96 & 68.1 \\
\hline \multicolumn{3}{|l|}{ Condición } \\
\hline Nombrado & 102 & 72.3 \\
\hline Contratado & 39 & 27.7 \\
\hline $\begin{array}{l}\text { Tiempo de servicio } \\
(\text { media } \pm \text { DS })\end{array}$ & \multicolumn{2}{|c|}{$17.7 \pm 8.1$} \\
\hline \multicolumn{3}{|l|}{ Turno } \\
\hline Mañana & 127 & 90.1 \\
\hline Tarde & 14 & 9.9 \\
\hline \multicolumn{3}{|l|}{ Burnout $($ media \pm DS $)$} \\
\hline $\begin{array}{l}\text { Agotamiento } \\
\text { emocional }\end{array}$ & \multicolumn{2}{|c|}{$13.4 \pm 8.9$} \\
\hline Despersonalización & \multicolumn{2}{|c|}{$2.2 \pm 3.3$} \\
\hline $\begin{array}{l}\text { Baja realización } \\
\text { personal }\end{array}$ & \multicolumn{2}{|c|}{$39.7 \pm 7.5$} \\
\hline \multicolumn{3}{|c|}{ Clima Social Familiar $($ media \pm DS $)$} \\
\hline Relación & 15. & \pm 2.0 \\
\hline Desarrollo & 29. & \pm 3.1 \\
\hline Estabilidad & 11. & \pm 2.4 \\
\hline
\end{tabular}

Las variables principales fueron el síndrome de burnout $y$ el clima social familiar, ambas cuantitativas. El burnout es un síndrome que implica síntomas de agotamiento emocional, despersonalización y baja realización personal en profesiones de interrelación con otras personas y que requiere la atención en los problemas que puedan presentar (4). Fue medido a través del Maslach Burnout Inventory (MBI) (4) el cual se evaluó a través de sus dimensiones: agotamiento emocional (nueve ítems), despersonalización (cinco ítems) y baja realización personal (ocho ítems). Consta de 22 ítems con un sistema de respuesta del 0 al 6. El mismo se interpreta a partir de la puntuación que se obtenga por cada dimensión: un puntaje alto indicaría un alto nivel en dicha dimensión.

El clima social familiar es el medio en el que una persona se relaciona con los integrantes de su familia y el impacto positivo que tiene en su comportamiento, emociones y cogniciones (12). Se midió a través de la escala de clima social familiar (FES) (12) que describe las relaciones entre los miembros de una familia, la importancia de su desarrollo y la estructura que la sostiene. La misma consta de noventa ítems y el rango de valores oscila entre 0 y 90 agrupados en diez sub-escalas que definen tres dimensiones: relación, desarrollo y estabilidad. Cada dimensión puede interpretarse como muy mala o muy buena, respectivamente.

En relación a las variables sociodemográficas consideradas fueron: la edad, variable cuantitativa discreta; tiempo de servicio, cuantitativa discreta; número de hijos, cualitativa politómica ordinal ( $\sin$ hijos, con un hijo y más de un hijo); estado civil, politómica nominal (soltera, conviviente, casada, separada, viuda, divorciada); turno, dicotómica nominal (mañana y tarde); relaciones personales, dicotómica nominal (con pareja estable, sin pareja estable) y condición, dicotómica nominal (nombrado, contratado). La recopilación de las mismas se realizó mediante una ficha de datos sociodemográfica.

Para el análisis de los datos se utilizó el paquete estadístico STATA 13. Con respecto al análisis descriptivo se calcularon frecuencias y porcentajes para las variables cualitativas y se determinó la media y desviación estándar para las cuantitativas. Se empleó la prueba de normalidad de Kolmogorov Smirnov para las variables numéricas. Para el análisis bivariado se usó $\mathrm{U}$ de Mann Whitney, Anova y Spearman. Asimismo, esta investigación fue aprobada por un Comité de Ética Institucional, igualmente se contempló la protección de los sujetos involucrados en el estudio. 


\section{RESULTADOS}

En la tabla 1 se muestra que las docentes presentaron una media de edad de 45.4, en cuanto al síndrome de burnout la dimensión de baja realización personal presentó el valor más alto con una media de 39.7, y respecto al clima social familiar se halló en la dimensión desarrollo una media de 29.1. Asimismo, el 58.2\% de docentes se encuentran casadas; el $70.3 \%$ refirió estar una relación estable y el $68.1 \%$ ha tenido más de un hijo. Respecto a su condición laboral se encontró que el $72.3 \%$ reportó que es nombrada, con una media de 17.7 años de servicio, desempeñándose el $90.1 \%$ en el turno mañana (ver tabla 1 ). $(\mathrm{p}=0.02)$ con la dimensión baja realización personal, ambas con una fuerza de correlación positiva muy débil (rho=0.17; rho=0.19). También la dimensión baja realización personal se relacionó con el estado civil ( $\mathrm{p}=0.05)$, donde la media más alta correspondió a las solteras $(\mathrm{m}=41.1)$. El resto de los datos se puede consultar en la tabla 2.

Por otra parte, se halló asociación significativa entre el turno y la dimensión de relación $(\mathrm{p}=0.05)$, en la cual el promedio más alto se reportó en las docentes que laboraban en el turno tarde $(\mathrm{m}=16.8)$.

Tabla 2. Asociación entre el síndrome de Burnout con el clima social familiar y las variables sociodemográficas

\begin{tabular}{|c|c|c|c|c|c|c|}
\hline & \multicolumn{6}{|c|}{ Burnout } \\
\hline & \multicolumn{2}{|c|}{$\begin{array}{c}\text { Agotamiento } \\
\text { Emocional }\end{array}$} & \multicolumn{2}{|c|}{ Despersonalización } & \multicolumn{2}{|c|}{$\begin{array}{c}\text { Baja Realización } \\
\text { Personal }\end{array}$} \\
\hline & media \pm DS & p-value & media \pm DS & p-value & media \pm DS & p-value \\
\hline \multicolumn{7}{|c|}{ Clima SocialFamiliar (Rho) } \\
\hline Relación & -0.17 & 0.04 & -0.06 & 0.45 & -0.06 & 0.45 \\
\hline Desarrollo & -0.12 & 0.17 & -0.13 & 0.11 & 0.17 & 0.04 \\
\hline Estabilidad & -0.05 & 0.54 & -0.09 & 0.30 & 0.19 & 0.02 \\
\hline Edad(Rho) & 0.09 & 0.27 & 0.07 & 0.38 & -0.11 & 0.19 \\
\hline Estado Civil & & 0.76 & & 0.48 & & 0.05 \\
\hline Soltero & $13.9 \pm 11.1$ & & $1.9 \pm 3.2$ & & $41.1 \pm 5.3$ & \\
\hline Conviviente & $13.6 \pm 9.1$ & & $3.6 \pm 3.8$ & & $36.7 \pm 12.2$ & \\
\hline Casado & $13.4 \pm 8.0$ & & $2.3 \pm 3.5$ & & $40.4 \pm 6.6$ & \\
\hline Separado & $9.2 \pm 9.2$ & & $0.7 \pm 1.4$ & & $40.0 \pm 9.8$ & \\
\hline Viudo & $11.5 \pm 2.1$ & & $1.0 \pm 1.4$ & & $36.0 \pm 5.6$ & \\
\hline Divorciado & $15.5 \pm 9.7$ & & $2.4 \pm 3.0$ & & $32.7 \pm 7.4$ & \\
\hline Relaciones Persor & ales & 0.39 & & 0.91 & & 0.54 \\
\hline $\begin{array}{l}\text { Con pareja } \\
\text { estable }\end{array}$ & $12.9 \pm 8.1$ & & $2.2 \pm 0.3$ & & $39.0 \pm 7.9$ & \\
\hline $\begin{array}{l}\text { Sin pareja } \\
\text { estable }\end{array}$ & $14.4 \pm 10.5$ & & $2.2 \pm 3.2$ & & $39.1 \pm 6.5$ & \\
\hline Número de hijos & & 0.86 & & 0.59 & & 0.85 \\
\hline Sin hijos & $13.1 \pm 9.1$ & & $1.3 \pm 2.1$ & & $38.9 \pm 7.7$ & \\
\hline Con 1 hijo & $14.1 \pm 10.3$ & & $2.2 \pm 3.2$ & & $40.2 \pm 5.9$ & \\
\hline Más de 1 hijo & $13.1 \pm 8.4$ & & $2.3 \pm 3.5$ & & $39.6 \pm 7.9$ & \\
\hline Condición & & 0.90 & & 0.79 & & 0.81 \\
\hline Nombrado & $13.3 \pm 8.6$ & & $2.2 \pm 3.2$ & & $39.8 \pm 7.6$ & \\
\hline Contratado & $13.5 \pm 9.5$ & & $2.3 \pm 3.6$ & & $39.5 \pm 7.1$ & \\
\hline Tiempo & & & & & & \\
\hline servicio (r) & 0.02 & 0.84 & -0.03 & 0.70 & -0.01 & 0.86 \\
\hline Turno & & 0.10 & & 0.18 & & 0.21 \\
\hline Mañana & $13.8 \pm 9.1$ & & $2.3 \pm 3.4$ & & $39.4 \pm 7.7$ & \\
\hline Tarde & $9.7 \pm 1.5$ & & $1.1 \pm 1.9$ & & $42.1 \pm 4.5$ & \\
\hline
\end{tabular}

Por otro lado, se encontró relación entre la dimensión relación y la dimensión de agotamiento emocional $(p=0.04)$, mostrando una fuerza de correlación negativa muy débil (rho=-0.17). Asimismo se evidenció relación de las dimensiones de desarrollo $(\mathrm{p}=0.04)$ y estabilidad
El resto de asociaciones se pueden ver en la tabla 3.

\section{DISCUSIÓN}

Las docentes que reportaron mayores niveles de agotamiento emocional presentaron menores 
niveles de relación dentro de la familia. Asimismo se evidenció que aquellas que presentaron baja realización personal mostraron bajos niveles en las dimensiones de estabilidad y desarrollo en el clima social familiar. Asimismo, las docentes que manifestaron baja realización personal fueron en su mayoría solteras. Por otra parte, se encontró asociación entre la variable turno con la dimensión de relación.
Las docentes que reportaron bajos niveles de desarrollo y estabilidad en el clima social familiar mostraron baja realización personal. El no poder soportar la presión y restar importancia a los procesos de desarrollo familiar conllevaría a la tendencia a evaluar su trabajo y evaluarse a sí mismas de manera negativa, evitando las relaciones interpersonales, profesionales y una baja producción laboral (14).

Tabla 3. Asociación entre el clima social familiar y las variables sociodemográficas

\begin{tabular}{|c|c|c|c|c|c|c|}
\hline & \multicolumn{6}{|c|}{ Clima social familiar } \\
\hline & \multicolumn{2}{|c|}{ Relación } & \multicolumn{2}{|c|}{ Desarrollo } & \multicolumn{2}{|c|}{ Estabilidad } \\
\hline & media \pm DS & p-value & $\operatorname{media} \pm \mathrm{DS}$ & p-value & $\operatorname{media} \pm \mathrm{DS}$ & p-value \\
\hline Edad (Rho) & 0.01 & 0.95 & 0.12 & 0.16 & -0.13 & 0.12 \\
\hline Estado Civil & & 0.33 & & 0.83 & & 0.67 \\
\hline Soltero & $15.5 \pm 2.3$ & & $28.9 \pm 4.4$ & & $11.6 \pm 2.3$ & \\
\hline Conviviente & $16.4 \pm 2.1$ & & $27.9 \pm 4.7$ & & $11.2 \pm 2.8$ & \\
\hline Casado & $15.8 \pm 2.0$ & & $29.3 \pm 3.9$ & & $11.9 \pm 2.4$ & \\
\hline Separado & $14.8 \pm 1.8$ & & $28.4 \pm 2.9$ & & $11.2 \pm 2.5$ & \\
\hline Viudo & $14.5 \pm 0.7$ & & $29.5 \pm 0.7$ & & $12.5 \pm 0.7$ & \\
\hline Divorciado & $16.5 \pm 1.3$ & & $30.0 \pm 3.8$ & & $13.0 \pm 2.6$ & \\
\hline \multicolumn{7}{|l|}{ Relaciones } \\
\hline Personales & & 0.25 & & 0.83 & & 0.47 \\
\hline Con pareja estable & $15.9 \pm 2.0$ & & $29.2 \pm 4.2$ & & $11.9 \pm 2.4$ & \\
\hline Sin pareja estable & $15.5 \pm 2.0$ & & $29.0 \pm 3.5$ & & $11.7 \pm 2.3$ & \\
\hline Número de hijos & & 0.67 & & 0.47 & & 0.76 \\
\hline Sin hijos & $15.8 \pm 2.1$ & & $29.6 \pm 3.6$ & & $11.4 \pm 3.0$ & \\
\hline Con 1 hijo & $15.5 \pm 2.2$ & & $28.4 \pm 4.1$ & & $11.6 \pm 2.6$ & \\
\hline Más de 1 hijo & $15.9 \pm 2.0$ & & $29.3 \pm 4.0$ & & $11.9 \pm 2.3$ & \\
\hline Condición & & 0.18 & & 0.36 & & 0.82 \\
\hline Nombrado & $15.9 \pm 2.0$ & & $29.3 \pm 3.8$ & & $11.8 \pm 2.2$ & \\
\hline Contratado & $15.4 \pm 2.1$ & & $28.6 \pm 4.5$ & & $11.7 \pm 2.8$ & \\
\hline Tiempo de servicio & & & & & & \\
\hline (r) & 0.01 & 0.91 & 0.20 & 0.02 & 0.01 & 0.87 \\
\hline Turno & & 0.05 & & 0.97 & & 0.88 \\
\hline Mañana & $15.7 \pm 2.0$ & & $29.1 \pm 3.9$ & & $11.8 \pm 2.3$ & \\
\hline Tarde & $16.8 \pm 1.8$ & & $29.1 \pm 4.5$ & & $11.5 \pm 3.5$ & \\
\hline
\end{tabular}

Las docentes con mayor agotamiento emocional poseen menores niveles en el clima social familiar. Esto es también confirmado en otros estudios $(6,11)$. El desgaste emocional en las docentes y su asociación con la relación dentro de la familia se explicaría mediante la sobrecarga que presentan, ya que el maestro cumple su función como agente socializador en diversos ámbitos: la escuela, la familia y el entorno laboral en el que se desenvuelve $(6,11)$. La presencia de estresores extra laborales tales como la relación intrafamiliar, dificultades de salud personal o familiar, y la sobre exigencia en sus hogares para cubrir los espacios de tiempo que pasan fuera del hogar debido al trabajo conllevarían a un mayor desgaste emocional (13).
La dimensión estabilidad trata de la información sobre la estructura, organización y grado de control que ejercen unos miembros sobre otros dentro de su desarrollo familiar (14). Por ello, cuando los docentes perciben el ambiente familiar como estimulante y con las condiciones necesarias para desarrollarse personalmente en diferentes aspectos de su vida, visualizan su entorno familiar con una buena estructura y organización. Contrariamente, la inadecuada estabilidad percibida en la familia sería un limitante en cuanto al desarrollo personal (14).

Por otro lado, las docentes que manifestaron baja realización personal poseen el estado civil de solteras. Se afirma en otro estudio que los maestros 
solteros experimentan mayor burnout, cansancio emocional y despersonalización (15). Una posible explicación está conectada a la relevancia de las relaciones familiares con la pareja o con los hijos, consideradas como un factor importante en la realización personal del individuo (15). Respecto a ello Maslach (7) refiere que el tener hijos se ve como un factor protector frente al burnout, puesto que la experiencia en cuanto a la resolución de problemas de un padre deviene de la maduración y del soporte emocional que podría brindar el ámbito familiar. Asimismo, llevar a casa cuestiones de trabajo se considera como factor de riesgo y acentúa la fuente de estrés pues de alguna manera no refuerzan las adecuadas relaciones entre los integrantes de la familia (15), perjudicando la interacción y por ende el desarrollo personal de cada uno de sus miembros.

Con respecto al turno de trabajo de las docentes se asoció con la dimensión relación del clima social familiar. Este resultado es similar a lo hallado en un estudio, donde el grado de comunicación, libre expresión y grado de interacción conflictiva, dentro de la familia se asocian con el turno de trabajo en que se encuentran desempeñando las docentes (14). Dentro de la relación interdependiente entre los miembros de una familia se encuentran inmersas diversas conductas, acciones incluso decisiones en la que los integrantes son partícipes; esto hace que las docentes también estén pendientes de sus familiares dentro de los horarios que disponen, ya que muchas de ellas cumplen un rol activo dentro de sus estructuras hogareñas como el rol materno, rol de hijas, hermanas o tías y es el tiempo que comparten con ellos lo que hace también posible el relacionarse con sus familias (14). Asimismo, la familia como unidad biopsicosocial y símbolo de unión estable debe ser la que brinda y cumple funciones relevantes para el desarrollo integral de cada uno de sus miembros, por ello es que se toma en cuenta el tiempo que se les otorga durante su desenvolvimiento vital (16), lo anterior dependerá del tiempo dedicado al trabajo, el horario del mismo y las horas fuera del hogar.
En cuanto a las limitaciones del estudio se considera que al ser de diseño transversal no se puede observar el comportamiento de las variables a lo largo del tiempo. Asimismo, al ser de tipo correlacional no se puede identificar la relación causa-efecto entre las variables principales. Por otro lado, las respuestas a las pruebas psicológicas pudieron ser influenciadas por la deseabilidad social. Se recomienda considerar otras variables sociodemográficas como tipo de familia, trabajos adicionales y el tipo de institución en la que trabaja (pública o privada).

\section{CONCLUSIONES}

Los resultados muestran que los niveles de desgaste emocional se asocian con los niveles de interacción dentro de la familia. Asimismo, se encuentra que la tendencia a evaluar el trabajo y evaluarse a sí mismo de manera negativa, evitando las relaciones interpersonales y profesionales teniendo baja producción sin poder soportar la presión se asocia con la importancia de procesos de desarrollo familiar, también con la información sobre la estructura, organización y grado de control que ejercen unos miembros de la familia sobre otros y de igual forma se vincula con el estado civil. Por último, se evidencia asociación entre la dimensión de relación del clima familiar (grado de comunicación, libre expresión, grado de interacción conflictiva) con el turno de trabajo.

Teniendo en cuenta estos hallazgos se recomienda la elaboración y ejecución de talleres de capacitación para la población evaluada en cuanto a estrategias de afrontamiento para el desarrollo de habilidades de hacer frente a los síntomas físicos, emocionales y psíquicos del síndrome de burnout. A los representantes educativos se recomienda considerar los resultados para contribuir con el mantenimiento y fortalecimiento del clima social familiar de las docentes de educación inicial. Es importante la implementación de programas preventivos promocionales como talleres $\mathrm{y}$ conferencias que potencien habilidades sociales, de autorrealización, de autoeficacia y estrategias de recreación a fin de mejorar los niveles del desarrollo integral de las docentes.

\section{REFERENCIAS BIBLIOGRÁFICAS}


1. Díaz, F.; López, A. y Varela, M. T. Factores asociados al síndrome de burnout en docentes de colegios de la ciudad de Cali, Colombia. Universitas Psychologica. 2012. 11(1),217-227.

2. Leka, S., Griffiths, A. y Cox, T. La 7 Organización del trabajo y el estrés. Estrategias sistemáticas de solución de problemas para 8 . empleados, personal directivo y representantes sindicales. Serie protección de la salud de los trabajadores $n^{\circ} 3$. Organización Mundial de la Salud: Francia; 2004. Recuperado de http://www.who.int/occupational_ health/publications/pwh3sp.pdf?ua 9 . $=1$

3. Organización Internacional del Trabajo. Estrés en el trabajo. Un reto colectivo. Ginebra; 2016: OIT. Recuperado de http://www.ilo.org/public/libdoc/il o/2016/490658.pdf

4. Maslach, C. y Jackson, S. MBI Inventario "Burnout" de Maslach Síndrome del quemado por estrés laboral. [Internet]. TEA ediciones: Madrid;

1997.

5. Cuenca, R. y O'Hara, J. El estrés en los maestros: percepción y realidad. Estudio de casos en Lima metropolitana. Lima; 2006

6. Cumpa, F. y Chávez, P. Síndrome de burnout en docentes del nivel primario de las instituciones educativas estatales del distrito de Chiclayo; Agosto, 2015. UMB. Disponible

en

http://repositorio.umb.edu.pe/hand le/UMB/21

Rottencacher, J. Una mirada a la salud mental desde la opinión de los peruanos. Boletín $\mathrm{N}^{\circ} 150$.

Ministerio de Educación del Perú. Indicadores de profesores titulados nivel inicial. [Internet]. ESCALE Unidad de Estadística Educativa; 2015. Disponible en: http://escale.minedu.gob.pe/tenden cias

\section{Murillo, J. Modelos Innovadores} en la formación inicial docente. Santiago de Chile; 2006.

10. Ministerio de Educación del Perú. Lineamientos para el seguimiento y control de la labor efectiva de trabajo docente en las instituciones educativas públicas. Artículo $4^{\circ}$. [Internet]. [Consultado 29 Nov 2018]. Disponible en: http://www.minedu.gob.pe/normat ividad/reglamentos/LineamSegContTrabDocente.php.

11. Arias, W. y Jiménez, N. Síndrome de burnout en docentes de educación básica regular de Arequipa. Revista Educación. $2013 . \quad 21(42), 53-76$.

12. Pezúa, M. Clima social familiar y su relación con la madurez social del niño(a) de 6 a 9 años.
[Internet]. [Tesis de Maestría]: Universidad Nacional Mayor de San Marcos; 2012. Disponible en: http://cybertesis.unmsm.edu.pe/ha ndle/cybertes is/3304

13. Oramas, A. Estrés laboral y síndrome de burnout en docentes cubanos de enseñanza primaria. [Internet]. [Tesis de grado]: Ministerio de salud pública de Cuba; 2013. Disponible en: http://tesis.sld.cu/index.php?P=Ful IRecord \&ID=287

14. Guevara, B. Nivel del Clima Social Familiar de los Docentes de las Instituciones Educativas San Antonio, San Juan Bautista y Luis Paredes Maceda. Yamango. [Internet]. [Tesis de Grado]: Universidad Católica Los Ángeles de Chimbote; 2016. Disponible en: http://repositorio.uladech.edu.pe/h andle/123456789/107

15. Moriana, J. y Herruzo, J. Estrés y burnout en profesores. [Internet]. International Journal of Clinical and Health Psychology. 2004. 4(3),597-621.

16. Mikuli, I. y Casullo, G. Algunas consideraciones acerca del concepto de clima social y su evaluación. [Internet]. Universidad de Buenos Aires. Facultad de Psicología. Disponible en: http://www.bibliopsi.org/docs/carr eras/obligatorias/CFG/psicometric as/mikulic/FICHA\%204.pdf 